\title{
Análisis bibliométrico de la situación de las mujeres investigadoras de Ciencias Sociales y Jurídicas en España
}

\author{
Daniel Torres-Salinas*, Ana M. Muñoz-Muñoz ${ }^{\star *}$, \\ Evaristo Jiménez-Contreras ${ }^{\star *}$
}

Resumen: Se presenta un estudio bibliométrico cuyo objetivo es analizar la productividad y la citación de las investigadoras en las Ciencias Sociales y Jurídicas en España en el marco de las revistas científicas nacionales. Como fuente de información se han empleado los rankings de investigadores/as presentes en el Índice de Impacto de las Revistas Españolas de Ciencias Sociales (INRECS) y el Índice de Impacto de las Revistas Españolas de Ciencias Jurídicas (INRECJ). El estudio analiza 11 disciplinas científicas diferentes y la muestra final de investigadores/as extraídos de dichos rankings ha sido de 3.370 de las que el 30\% eran mujeres. Para estudiar las diferencias de géneros se han seleccionado y analizado cuatro variables: el número de trabajos publicados, el número de citas obtenidas, número de citas internacionales y el promedio de citas. Los resultados muestran como las mujeres en las 11 áreas analizadas tienen una menor productividad así como un menor número de citas bruto. Por otro lado las mujeres en diversas áreas sí obtienen un mayor número de citas internacionales y en casi todas alcanzan promedios de citas mayores. Sin embargo aún es escasa la presencia de las mujeres en los rankings y en la élite científica española.

Palabras clave: indicadores bibliométricos, estudios de género, investigadoras, Ciencias Sociales, Ciencias Jurídicas.

\section{Bibliometric analysis of the situation of female researchers in the fields of social sciences and law in Spain}

Abstract: This paper presents a bibliometric study analyzing the productivity and citations offemale researchers in the fields of social sciences and law in Spain, within the context of Spanish scientific journals. The study was based on the rankings of researchers

* Centro de Investigación Médica Aplicada, Universidad de Navarra. Pamplona, España. Correo-e: torressalinas@gmail.com.

** Departamento de Biblioteconomía y Documentación, Universidad de Granada, Granada, España.

Recibido:08-07-2010; 2. ${ }^{a}$ versión:16-09-2010; aceptado:05-10-2010. 
included in the Indice de Impacto de las Revistas Españolas de Ciencias Sociales (INRECS) and Índice de Impacto de las Revistas Españolas de Ciencias Jurídicas (INRECJ). Eleven different scientific disciplines were analyzed and the final sample consisted of 3,370 researchers, of whom $30 \%$ were women. To study gender differences four variables were selected and analyzed: number of papers, number of citations, number of international citations and citation average. The results show that women in the 11 areas studied have both a lower productivity and a lower number of citations. On the other hand, in some areas women do receive a greater number of international citations and in almost all areas achieve higher citation averages. However the presence of women in the rankings and among the Spanish research elite is still small.

Keywords: Bibliometric Indicators, Gender Studies, Women Researchers, Social Sciences, Law.

\section{Introducción}

En la actualidad existe un creciente interés en España en mejorar la situación de las mujeres en diferentes ámbitos profesionales entre los que se incluyen los científicos y académicos. Este interés se ha manifestado legislativamente a través del desarrollo de una serie de políticas para equiparar las condiciones de las mujeres a la de los hombres. A nivel general destaca la promulgación de la Ley Orgánica 3/2007, de 22 de marzo, para la igualdad efectiva de mujeres y hombres y más específicamente la Ley Orgánica 4/2007, de 12 de abril, por la que se modifica la Ley Orgánica 6/2001, de 21 de diciembre, de Universidades. En ésta última se señalan como objetivos la búsqueda de paridad en los órganos de representación y una mayor participación de la mujer en los grupos de investigación. Por ello, en estos momentos, visibilizar numéricamente y conocer el estado de la mujeres en el ámbito científico es importante ya que como afirma Huyer y Westholm (2002) "sin datos no hay visibilidad; sin visibilidad no hay prioridad". En este sentido en los últimos diez años se han elaborado diagnósticos sobre la circunstancias de las mujeres en diversos ámbitos de la ciencia española (Alcalá y otros, 2005; Arranz, 2004; García de Cortázar y otros, 2006; García de León y García de Cortázar, 2001; Muñoz-Muñoz, 2005, 2006, Guerrero-Bote y otros, 2009). La mayor parte de estas aportaciones, siguiendo el modelo de estudios europeos (European Commission, 2000, 2006), se realizaron con el objetivo de contribuir a analizar los factores y circunstancias relacionadas con la escasa participación y el liderazgo de la mujer en el sistema de investigación científica, situaciones que se han explicado a través de los denominados efectos tijera y cañería con fugas (leaky pipe) (European Commission, 2009). Junto a los estudios señalados hemos de mencionar como herramienta para conocer el estado de las mujeres en la ciencia los estudios bibliométricos.

En la literatura bibliométrica que aplica una perspectiva de género se puede comprobar que gran parte de estos análisis se realizan con el propósito general de identificar posibles desigualdades entre hombres y mujeres. A nivel metodológico se centran en analizar o bien la productividad de las mujeres o bien su 
impacto en comparación con sus colegas masculinos. En este contexto, a nivel internacional encontramos el análisis del Council of Scientific and Industrial Research (CSIR) de Nueva Delhi que concluye que no existen diferencias en la productividad de los científicos masculinos y femeninos (Gupta y otros, 1999), conclusiones similares a las que se alcanzan en estudios posteriores (Palomba y Menniti, 2001; Lewison, 2001). Sin embargo distintos estudios sobre el profesorado universitario realizados en otros países como Italia o Croacia si identifican contrastes significativos en la productividad de hombres y mujeres. En el caso italiano son mejores los resultados de los hombres, aunque existen determinados sectores científicos en los que las mujeres se muestran más productivas (Abramo y otros, 2009); en el croata incluso las mujeres más productivas publican menos que sus homólogos masculinos (Prpic, 2002).

A nivel nacional, en los estudios sobre la producción científica desagregada por sexo realizados sobre el Consejo Superior de Investigaciones Científicas (CSIC), se determina que no hay diferencias relevantes en el número de publicaciones y su calidad entre hombres y mujeres del mismo nivel académico (Alcalá y otros, 2005). Sin embargo en el propio CSIC, en el área de Ciencias Materiales, se da el caso de que las mujeres son menos productivas que los hombres, si bien las diferencias casi no existen cuando se trata de la misma categoría profesional (Mauleón y Bordons, 2006). En un estudio comparativo de las áreas de Recursos Naturales y Química de la misma institución los resultados tampoco mostraron diferencias importantes entre ambos géneros pero si destacan que los investigadores con mayor producción eran en su mayoría hombres (Bordons y otros, 2003).

En general, una característica común a casi todos los trabajos bibliométricos referenciados es su limitación a muestras de investigadores relativamente reducidas por lo que sus resultados tal vez no sean representativos del conjunto de la población investigadora de un país o una disciplina. Este uso de muestras pequeñas se produce porque los estudios bibliométricos que introducen la variable de género son difíciles de ejecutar debido a diferentes factores. En primer lugar habría que señalar la falta de normalización en el nombre de los autores que existe en las bases de datos internacionales como la Web of Science o Medline; dicho problema se agrava aún más en el caso español ya que dichas bases de datos emplean prácticas de indización adaptadas a los nombres ingleses por lo que un autor puede aparecer indexado bajo múltiples formas (Ruíz-Pérez y otros, 2003). En segundo lugar y unido al anterior se plantean bastantes dificultades a la hora de reconocer el sexo de los firmantes (Russell, 2003), ya que al indexar solamente la inicial del nombre y no desarrollarlo de forma completa provoca que sea difícil saber si los trabajos han sido firmados por un hombre o una mujer. Por ello la mayor parte de los análisis bibliométricos de género se circunscriben a entornos institucionales en los que se controlan los datos administrativos del personal investigador así como su producción científica. Ésta es un de las principales razones por las que a nivel nacional aún no contamos con ningún estudio bibliométrico realizado con una perspectiva de género que analice de 
forma global la ciencia española. Aunque un estudio de estas características es de difícil ejecución en la actualidad contamos con INRECS e INRECJ cuya explotación puede ayudar a dilucidar la situación de la mujer en la ciencia española, al menos en el ámbito de las Ciencias Sociales y Jurídicas.

\section{Objetivos}

El objetivo general de este trabajo es analizar la representación, la productividad y el impacto de los artículos publicados por las mujeres en las Ciencias Sociales y Jurídicas españolas en comparación con los hombres. Se pretende comprobar con ello, a través de diversos indicadores bibliométricos, si existen diferencias de género. Más específicamente podemos establecer los siguientes objetivos de investigación:

1. Estudiar la representación de las mujeres en las principales áreas de las Ciencias Sociales.

2. Establecer las diferencias entre hombres y mujeres en función del número de trabajos que publican y el número de citas que éstos reciben.

3. Analizar la presencia de las mujeres en la élite científica, entendida ésta como el conjunto de investigadores/as con unos indicadores de calidad científica más altos.

\section{Material y métodos}

Para obtener una muestra amplia de investigadores se seleccionaron como fuente de información los siguientes productos elaborados por el Grupo Evaluación de la Ciencia y la Comunicación Científica (Grupo EC3): INRECS-Índice de Impacto de las Revistas Españolas de Ciencias Sociales INRECJ-Índice de Impacto de las Revistas Españolas de Ciencias Jurídicas (Jiménez-Contreras y otros, 2008). Dichos productos indexan conjuntamente en su edición de 2008 un total de 810 revistas científicas españolas para un total de 10 disciplinas de Ciencias Sociales y 11 de Derecho con el objeto de calcular su Índice de Impacto. Además de evaluar revistas científicas a partir de los datos obtenidos de las mismas, tanto INRECS como INRECJ, presentan rankings de investigadores agrupados por disciplina donde se calculan un total de ocho indicadores bibliométricos de producción e impacto (Figura 1). Precisamente han sido los investigadores presentes en estos rankings los seleccionados como muestra para este estudio. La condición esencial por la que el personal investigador aparece en estos listados es que al menos hayan publicado un trabajo y cuenten con un determinado número de citas que varía según la disciplina. Respecto a la cobertura cronológica que abarcan los rankings para el cálculo de los distintos indicadores es el período 1996-2008 para el caso de todo el personal investigador presente en INRECS y 
2001-2008 en el caso de INRECJ. Aunque para ambos productos se empleen coberturas distintas esta situación no afecta a este estudio, ya que el objetivo del mismo no es tanto comparar unas disciplinas contra otras sino observar las diferencias entre hombres y mujeres a nivel intradisciplinar.

\section{FIGURA 1}

Ejemplo de los rankings de investigadores empleados en INRECS/INRECJ

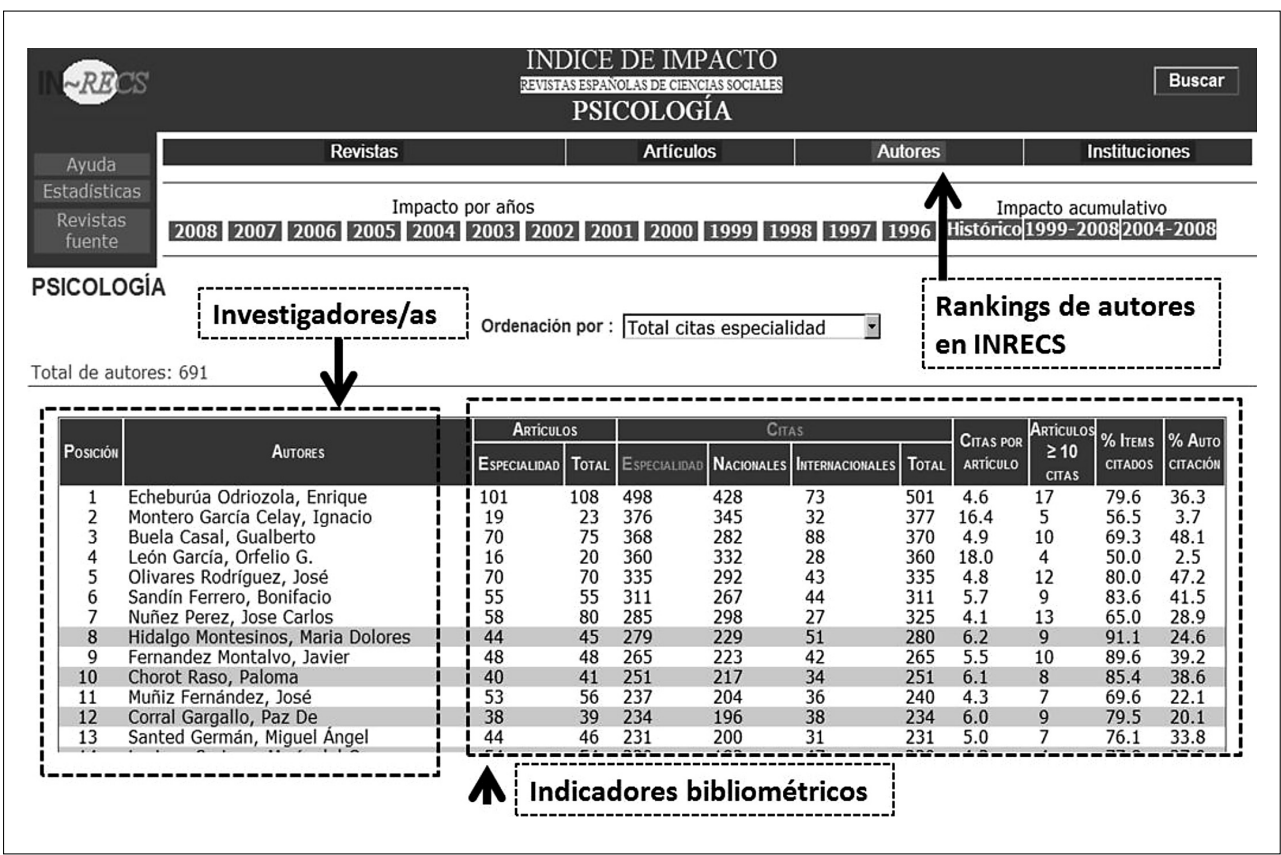

En cuanto a la fuente de información seleccionada (INRECS; INRECJ) cabe plantearse su idoneidad para alcanzar los objetivos de este estudio. Sin embargo la única alternativa son las bases de datos internacionales de carácter multidisciplinar, entre las que destaca el Social Sciences Citation Index (SSCI); dicha fuente no es del todo apropiada ya que en gran parte de las Ciencias Sociales españolas las revistas nacionales suelen ser el medio preferente de publicación, siendo escaso el número de autores que publican sistemáticamente en revistas extranjeras, a excepción de áreas como la Psicología o parte de la Economía. Según Delgado y otros (2005) tan solo el 10\% de las Ciencias Sociales españolas están representadas en SSCI. Como ejemplo, podemos señalar áreas con una buena cobertura en INRECS/INRECJ pero escasamente representadas en SSCI en los últimos diez años (2000-2009): Derecho (114 artículos), Urbanismo (204 artículos), Comunicación (207 artículos) y Ciencia Política (213 artículos). Podemos concluir al respecto que las Ciencias Sociales y Jurídicas españolas tienen todavía 
poco grado de internacionalización y que una fuente de carácter nacional como INRECS/INRECJ es más adecuada, o al menos la única disponible, para este tipo de estudios.

Seleccionada la fuente de información se descargaron los rankings de investigadores con sus respectivos indicadores (Figura 1) para cada una de las disciplinas y se procedió a la normalización de nombres de los autores. Aunque los productos empleados ya presentan un importante grado de normalización y la mayoría de las autorías figuran bajo una sola denominación en casos puntuales esta situación no se produce. En segundo lugar, se eliminaron de los listados las autorías extranjeras, que pese a figurar en los rankings no forman parte de ninguna universidad u OPI español. Asimismo, en ocasiones, un investigador puede estar presente en dos disciplinas al mismo tiempo, para evitar este problema dichos investigadores y su producción científica fueron asignados únicamente a aquellas disciplinas en las que tenían una mayor producción científica. En el caso de las 11 áreas que conforman INRECJ se unificaron como una única disciplina (Derecho). Una vez ejecutado el proceso de normalización el total de investigadores que conformaron la muestra final fue de 3.397, la distribución de este conjunto por disciplina se muestra en el Figura 2. Las disciplinas con un mayor número de investigadores/as son Derecho y Psicología con el 37\% y el $17 \%$ de la muestra mientras que Antropología Social y Urbanismo solo representan el $1 \%$.

\section{FIGURA 2}

Origen disciplinar de la muestra del personal investigador empleada. Período 2001-2008 en Derecho y 1996-2008 en el resto de disciplinas

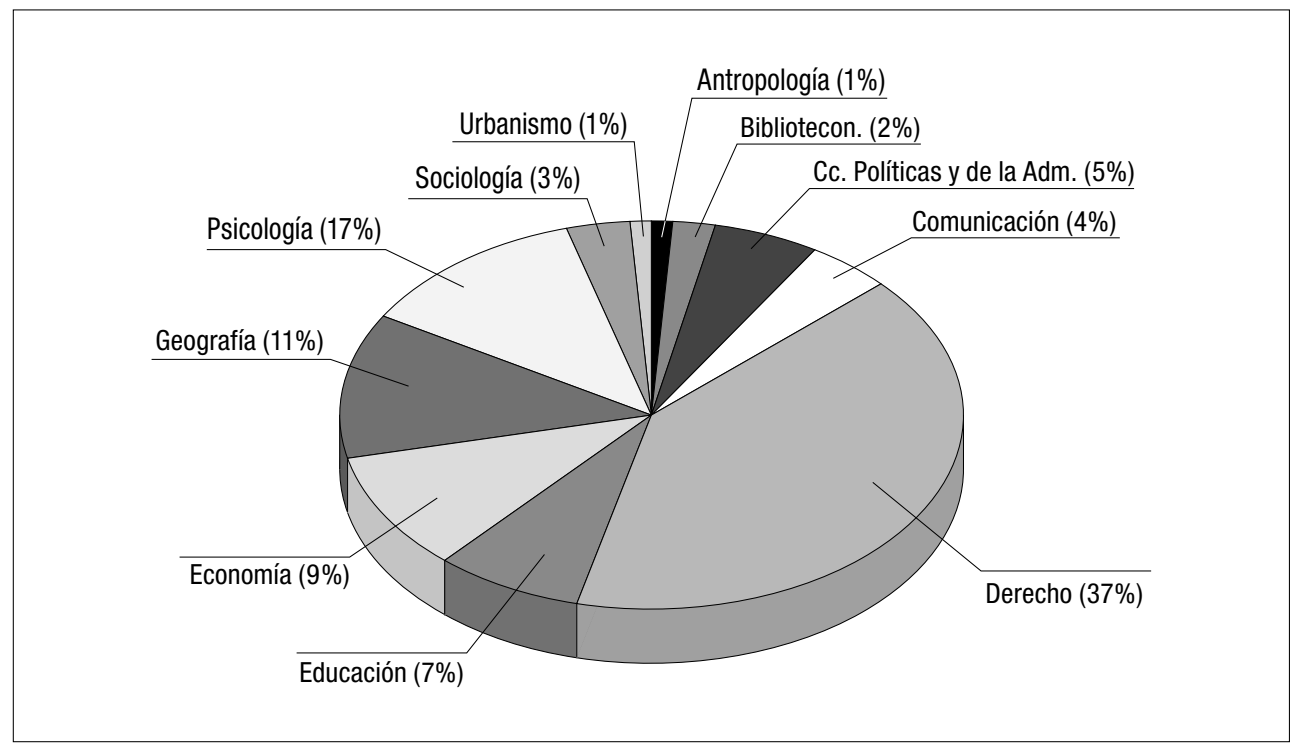


Del total de indicadores que nos ofrecen los rankings tan sólo se han seleccionado cuatro:

- Número de trabajos publicados en revistas científicas españolas. Es el número de trabajos publicados por el personal investigador en las revistas españolas indexadas en INRECS/INRECJ. Para cada autor se contabilizan todos los trabajos que hayan firmado, independientemente de que éstos sean en colaboración o de una sola coautoría.

- Número total de citas recibidas. Es el número de citas recibidas por el personal investigador en los trabajos publicados en revistas indexadas en INRECS/INRECJ. Se contabilizan en este caso tanto citas recibidas desde revistas fuente INRECS/INRECJ como de revistas internacionales.

- Número de citas internacionales. Es el número bruto de citas que han recibido los trabajos publicados en revistas indexadas en INRECS/INRECJ desde revistas científicas internacionales. En este caso se consideran como revistas científicas internacionales las indexadas en Web of Science.

- Promedio de citas por trabajo. Para el cálculo de este indicador se tiene en cuenta el Número total de citas recibidas y el Número de trabajos publicados en revistas científicas españolas.

Para obtener indicadores complementarios que permitieran contextualizar los resultados se utilizaron otros datos como los incluidos en las series Estadística para la Enseñanza Universitaria en España: Personal docente de los centros propios de las Universidades Públicas por Área de conocimiento, Sexo y Categoría del Instituto Nacional de Estadística (INE) (2010). De la página web del INE se descargaron las series relativas a todos cursos académicos comprendidos entre el curso 1998/1999 y 2007/2008. Con dichas series se ha obtenido el promedio de profesores y profesoras anual que trabajan en cada una de las áreas. Estas series estadísticas clasifican al personal docente de las universidades en 210 áreas de conocimiento por lo que es posible hacer una equivalencia entre áreas INE y áreas INRECS/INRECJ. Por otro lado para poder comparar estos datos con los obtenidos en INRECS se han empleado las series comprendidas entre los cursos 1998/1999 y 2007/2008, sin embargo, en el caso de INRECJ, al ser diferente la cobertura, los datos se han limitado a las series comprendidas entre los cursos 2001/2002 y 2007/2008.

Igualmente se descargó del sitio web de la Comisión Nacional Evaluadora de la Actividad Investigadora (CNEAI) un fichero Excel con la distribución de los sexenios por área y sexo incluido en su Informe 2009 sobre los resultados de las evaluaciones de la CNEAI (CNEAI, 2009). CNEAI es una de las agencias españolas de evaluación de la actividad científica y afecta al profesorado funcionario universitario y a las escalas científicas del CSIC. La CNEAI realiza convocatorias anuales y los solicitantes voluntariamente someten a evaluación cinco publicaciones realizadas en un período mínimo de 6 años; la evaluación positiva supone la obtención de un sexenio y los solicitantes a lo largo de su carrera pueden 
obtener hasta un máximo de 6. La obtención de un tramo supone un complemento retributivo y es una de las fórmulas fundamentales del reconocimiento científico de nuestro sistema de ciencia y tecnología. (Fernández y otros, 2006).

\section{Resultados}

\subsection{Indicadores de producción e impacto}

En la tabla I se presenta los resultados generales. El número promedio de investigadores trabajando en las áreas analizadas es de 24.050 , de los que el 40\% son mujeres, este porcentaje varía entre el 16\% de Urbanismo y el 57\% de Biblioteconomía. La presencia de mujeres en los rankings de INRECS/INRECJ es de un $30 \%$, aunque por disciplinas existe una mayor variabilidad que va desde el 18\% de mujeres en el caso de Ciencia Política al 43\% de Psicología. Respecto a la aportación de las mujeres a la producción total y al número de citas las diferencias son mayores. En el caso de la producción existen áreas donde los trabajos firmados apenas llegan a representar el 15\%, como Urbanismo (9\%), Ciencia Política (11\%) o Sociología (13\%), mientras que, en el extremo opuesto, el valor máximo lo encontramos en Psicología donde el 36\% de los trabajos son firmados por mujeres. Para las citas, aunque con porcentajes sensiblemente superiores, se repite el mismo el patrón.

En el caso de la media de trabajos publicados por investigador en todas las áreas los hombres presentan valores más elevados (tabla II). Las disciplinas donde existe una mayor diferencia, llegando a ser más del doble son, Urbanismo (11,44-H; 4,46-M) y Sociología (17,52-H; 8,56-M). En el número de citas sólo encontramos dos disciplinas en las que las mujeres presentan valores más altos: Antropología (5,66-H, 6,60-M) y Comunicación (6,98-H; 7,25-M). Las citas internacionales son el indicador donde las mujeres logran los mejores resultados: de las 11 disciplinas analizadas, en 7 de ellas las investigadoras presentan valores más elevados. Finalmente, si tenemos en cuenta el promedio de citas en casi todas las disciplinas, a excepción de Biblioteconomía, las investigadoras tienen un promedio mayor con acusadas diferencias en Economía, Sociología y Urbanismo.

En la figura 3 se presenta la distribución de los 3.397 casos analizados para los cuatros indicadores. En todos los indicadores claramente hay una similitud entre hombres y mujeres desde los valores más bajos hasta la mediana de las distribuciones, hasta ese punto no existen diferencias significativas en la mayor parte de las disciplinas e indicadores. Sin embargo, existen un mayor número de casos extremos de hombres, lo que indica como éstos obtienen los valores más altos en los indicadores y en consecuencia alcanzan las posiciones más elevadas en los diferentes rankings. La situación mostrada se produce sobre todo para los indicadores de número de trabajos, número de citas y número de citas internacionales. En el caso de los promedios de citas existe un comportamiento más homogéneo en las zonas altas de la distribución. 


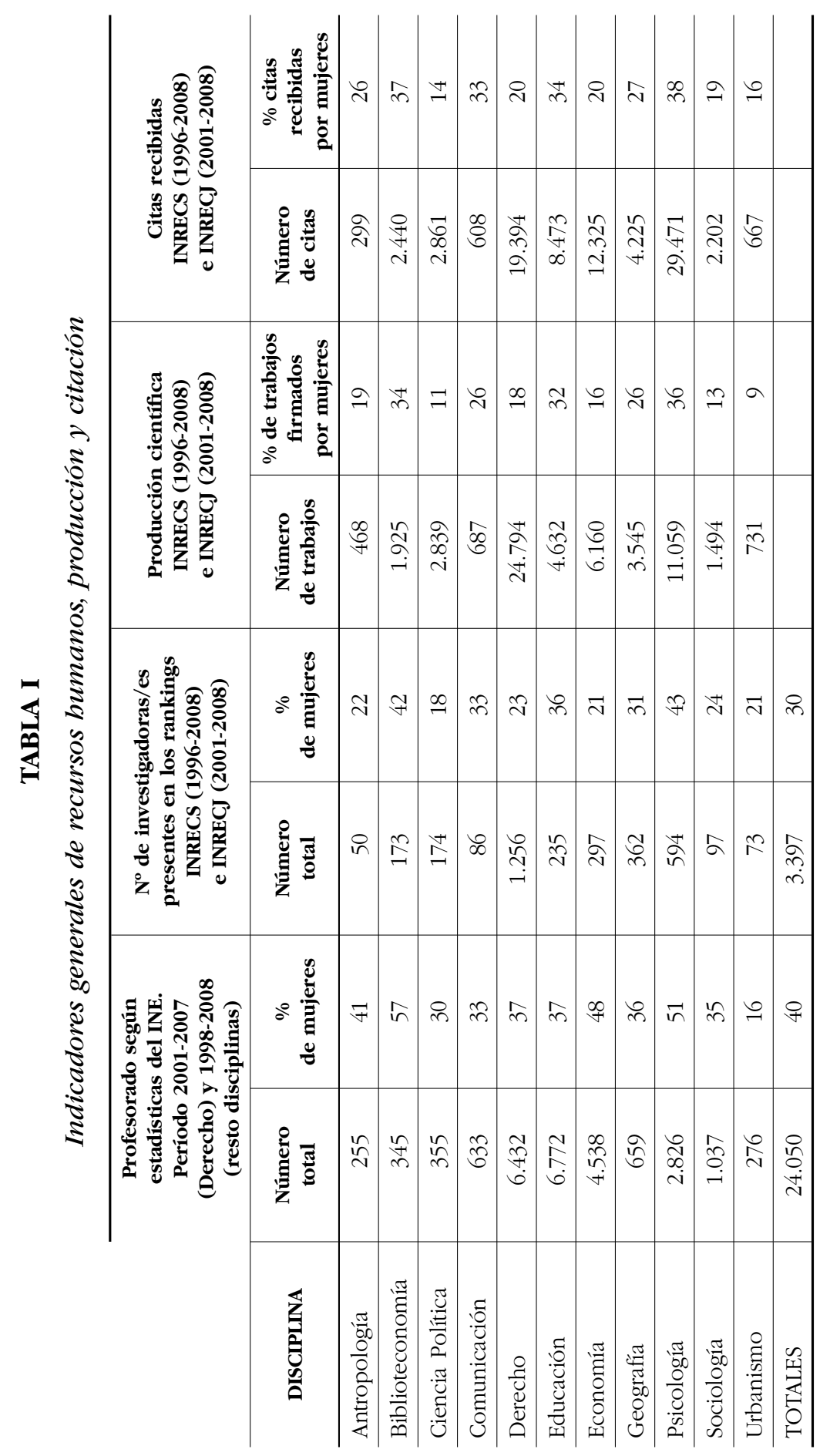




\begin{tabular}{|c|c|c|c|c|c|c|c|c|c|c|c|c|c|}
\hline \multirow{6}{*}{\multicolumn{2}{|c|}{ 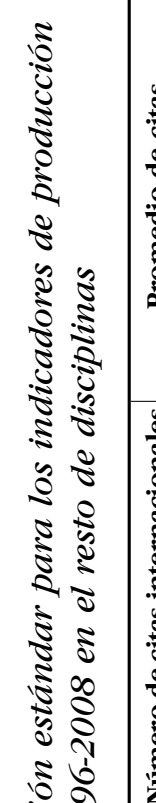 }} & 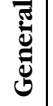 & \begin{tabular}{ll}
+1 & \multicolumn{1}{c}{} \\
2 & 0 \\
$\tilde{\sigma}$ & -
\end{tabular} & $\begin{array}{l}+1 \\
\stackrel{+}{0} \\
\stackrel{0}{0} \\
i\end{array}$ & 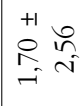 & $\underset{-}{\stackrel{+1}{0}} \stackrel{\sim}{\sim}$ & $\stackrel{+1}{8} \bar{\sigma}$ & $\begin{array}{l}+1 \\
\tilde{r}^{+} \\
\dot{n}^{-}\end{array}$ & 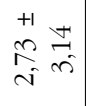 & 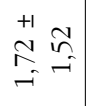 & $\begin{array}{ll}+1 & \infty \\
\alpha_{0} & 0 \\
n^{-1} & \text { in }\end{array}$ & 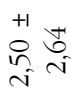 & $\stackrel{+1}{\vec{r}} \underset{-}{-}$ \\
\hline & & 离 & $\stackrel{+1}{\stackrel{n}{\approx}} \underset{-}{-}$ & 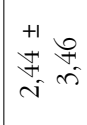 & $\begin{array}{ll}+1 & 0 \\
i & 8 \\
i & f\end{array}$ & 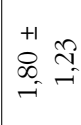 & $\begin{array}{ll}+1 & n \\
+ & \infty \\
-i & 0\end{array}$ & \begin{tabular}{l}
+1 \\
$\stackrel{+1}{2}$ \\
\multirow{*}{*}{}
\end{tabular} & $\begin{array}{ll}+1 & 0 \\
\infty & 6 \\
\infty & 0 \\
i & n\end{array}$ & $\begin{array}{l}+1 \\
+ \\
\stackrel{n}{=}\end{array}$ & 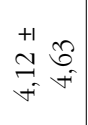 & 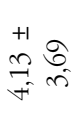 & $\begin{array}{ll}+1 & 0 \\
n & 2 \\
n^{2} & \text { in }\end{array}$ \\
\hline & & 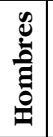 & $\begin{array}{ll}+1 & n \\
\infty & \approx \\
0 & 0\end{array}$ & $\begin{array}{l}+1 \\
\infty \\
\infty \\
i \\
i\end{array}$ & 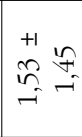 & 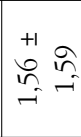 & $\begin{array}{l}+1 \\
\alpha \\
\sigma\end{array}$ & $\begin{array}{ll}+1 & 0 \\
\text { tr } & \infty \\
\text { iv } & 0\end{array}$ & $\begin{array}{ll}+1 & \infty \\
+ & \infty \\
\sigma^{2} & i \\
i & i\end{array}$ & $\stackrel{+1}{\stackrel{N}{\sim}} \underset{-}{\sim}$ & \begin{tabular}{ll|}
+1 & \\
$\sigma^{-}$ & $F$ \\
$r^{-}$ & -1
\end{tabular} & $\stackrel{+1}{\sigma} \underset{-}{\sigma}$ & $\stackrel{+1}{\stackrel{+}{2}} \underset{-1}{=}$ \\
\hline & & 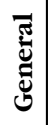 & 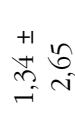 & $\begin{array}{l}+1 \\
\tilde{n} \\
\tilde{n} \\
\tilde{n}\end{array}$ & $\begin{array}{l}+1 \\
\tilde{n} \\
\tilde{n} \\
0\end{array}$ & 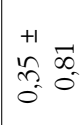 & $\begin{array}{ll}+1 & 0 \\
0 & \varrho \\
0 & 0\end{array}$ & 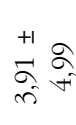 & 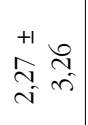 & $\begin{array}{ll}+1 & \circ \\
\stackrel{\infty}{=} & - \\
-\end{array}$ & $\left|\begin{array}{cc}+1 & \hat{2} \\
\infty & 1 \\
0 & 0 \\
\sigma & -\end{array}\right|$ & $\stackrel{+1}{\stackrel{n}{\circ}} \underset{-}{\infty}$ & $\begin{array}{l}+1 \\
\hat{n} \\
\hat{n}^{-} \\
0 \\
0\end{array}$ \\
\hline & & 离 & 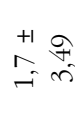 & \begin{tabular}{l}
+1 \\
\multirow{2}{*}{} \\
in \\
in
\end{tabular} & $\begin{array}{l}+1 \\
0 \\
0 \\
0 \\
0\end{array}$ & 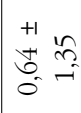 & 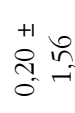 & $\begin{array}{ll}+1 & \approx \\
2 & \approx \\
\approx & f\end{array}$ & 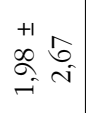 & $\begin{array}{ll}+1 & 0 \\
2 & = \\
0 & -1\end{array}$ & $\begin{array}{ll}+1 & 0 \\
0 & 0 \\
0 & \infty\end{array}$ & 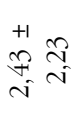 & $\begin{array}{l}+1 \\
0 \\
0 \\
0\end{array}$ \\
\hline & 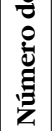 & 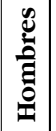 & $\begin{array}{l}+1 \\
\stackrel{+}{*} \\
\stackrel{+}{-}\end{array}$ & $\begin{array}{l}+1 \\
\Re \\
\sim_{+}^{*}\end{array}$ & $\begin{array}{l}+1 \\
i \approx \\
0\end{array}$ & $\begin{array}{ll}+1 & 1 \\
\text { సิ } & 0 \\
0 & 0\end{array}$ & $\begin{array}{ll}+1 & \infty \\
12 & 0 \\
0 & 0 \\
0 & 0\end{array}$ & $\begin{array}{l}+1 \\
\stackrel{2}{n^{2}} \\
\dot{m}^{-1}\end{array}$ & 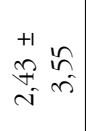 & $\begin{array}{ll}+1 & n \\
\vec{n} & i \\
-i & i\end{array}$ & $\begin{array}{ll}+1 & 0 \\
\approx & \sim \\
\approx & = \\
0 & =\end{array}$ & 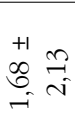 & 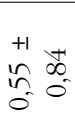 \\
\hline 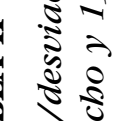 & I & 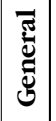 & $\begin{array}{l}+1 \\
\infty \\
\infty \\
\text { in }\end{array}$ & 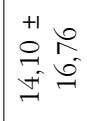 & \begin{tabular}{ll}
+1 & \multicolumn{1}{c}{} \\
8 & $\infty$ \\
$\sigma^{-}$ & in
\end{tabular} & 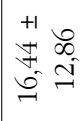 & 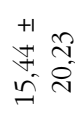 & 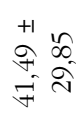 & 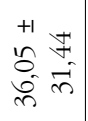 & 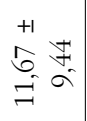 & 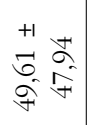 & $\begin{array}{l}+1 \\
\stackrel{8}{\circ} \\
\text { î } \\
\text { in }\end{array}$ & 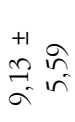 \\
\hline 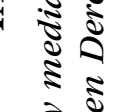 & $\begin{array}{l}u \\
0 \\
0 \\
\dot{0}\end{array}$ & $\frac{8}{0}$ & $\begin{array}{ll}+1 & \infty \\
0 & 8 \\
0 & \text { in }\end{array}$ & 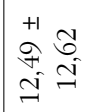 & 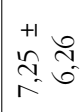 & 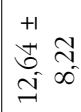 & 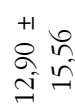 & $\begin{array}{ll}+1 & 0 \\
n & \sim \\
\alpha & \approx \\
\infty & \approx\end{array}$ & $\begin{array}{ll}+1 & n \\
\kappa & \infty \\
\alpha & \hat{v} \\
\approx & \approx\end{array}$ & $\begin{array}{ll}+1 & 0 \\
\infty & 0 \\
N & 1 \\
0 & 1\end{array}$ & \begin{tabular}{ll}
+1 & 0 \\
1 & 0 \\
$\infty$ & 0 \\
\multirow{2}{*}{} & $\sqrt[f]{-1}$
\end{tabular} & 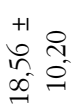 & 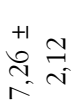 \\
\hline 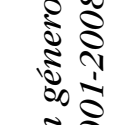 & z & 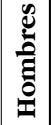 & $\begin{array}{l}+1 \\
\stackrel{0}{0} \approx \\
i n\end{array}$ & 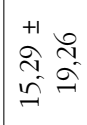 & $\begin{array}{ll}+1 & \text { in } \\
0 & 6 \\
\sigma_{0} & \text { in }\end{array}$ & 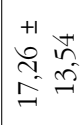 & $\begin{array}{l}+1 \\
\vec{ה} \\
\sigma_{-}^{-} \\
\vec{\sim}\end{array}$ & $\begin{array}{l}+1 \\
\stackrel{n}{n} \\
\vec{y} \\
\vec{n}\end{array}$ & $\begin{array}{ll}+1 & \vec{n} \\
\vec{N} & \vec{n} \\
\hat{n} & m\end{array}$ & 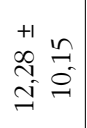 & 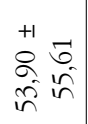 & 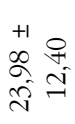 & $\begin{array}{l}+1 \\
\text { त̂ } \\
\sigma^{1}\end{array}$ \\
\hline $\begin{array}{l}0 \\
0 \\
0 \\
0 \\
0\end{array}$ & $\stackrel{8}{\circ}$ & 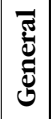 & $\begin{array}{ll}+1 & + \\
0 & 0 \\
2 & 0 \\
\sigma^{2} & 0\end{array}$ & $\begin{array}{l}+1 \\
\stackrel{N}{N} \\
\Rightarrow \\
\Rightarrow\end{array}$ & $\begin{array}{ll}+1 & 0 \\
0 & 2 \\
2 & n \\
n & n\end{array}$ & $\begin{array}{ll}+1 & \vec{n} \\
\vec{n} & \sigma^{2} \\
\sigma^{-1} & -\end{array}$ & 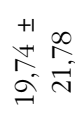 & 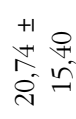 & 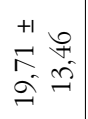 & \begin{tabular}{ll}
+1 & \multicolumn{1}{c}{} \\
2 & $b$ \\
$a$ & -
\end{tabular} & 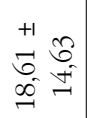 & $\begin{array}{l}+1 \\
\text { 요 } \\
\text { n. } \\
\text { n. }\end{array}$ & 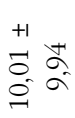 \\
\hline 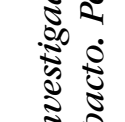 & $\begin{array}{l}\vec{J} \\
\bar{J} \\
0 \\
0 \\
0\end{array}$ & 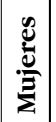 & $\begin{array}{l}+1 \\
2 \\
n\end{array}$ & $\begin{array}{l}n \\
n \\
+1 \\
a\end{array}$ & 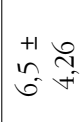 & $\begin{array}{ll}+1 & \\
8 & \infty \\
8 & \infty \\
0 & \infty\end{array}$ & $\begin{array}{ll}+1 & \approx \\
\infty & \approx \\
\infty & \ddots \\
\pm & =\end{array}$ & $\begin{array}{ll}+1 & \infty \\
\infty & 1 \\
0 & n \\
0 & = \\
-1 & =\end{array}$ & 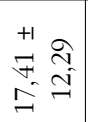 & $\begin{array}{cc}+1 & \\
\hat{\lambda} & \hat{0} \\
\infty^{-} & \text {in }\end{array}$ & 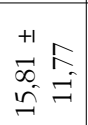 & 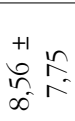 & $\begin{array}{ll}+1 & 0 \\
\text { o } & \text { in } \\
\forall & n\end{array}$ \\
\hline 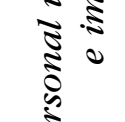 & 恶 & 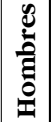 & $\begin{array}{l}+1 \\
\stackrel{+1}{2}= \\
\sigma_{0}\end{array}$ & 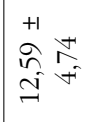 & $\begin{array}{l}+1 \\
N^{+} \\
\infty^{-}\end{array}$ & 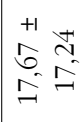 & 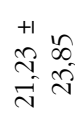 & $\begin{array}{ll}+1 & 1 \\
\vdots & 0 \\
\hat{a} & 0 \\
\vec{v} & 0\end{array}$ & 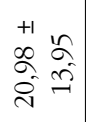 & 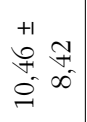 & $\begin{array}{ll}+1 & \infty \\
\hat{2} & 0 \\
0 & 0 \\
\tilde{n} & -1\end{array}$ & 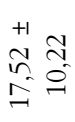 & 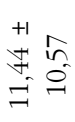 \\
\hline 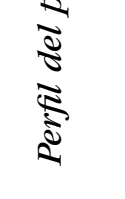 & & & $\begin{array}{l}\frac{\pi}{0} \\
0 \\
0 \\
0 \\
0 \\
0 \\
0 \\
0 \\
0\end{array}$ & 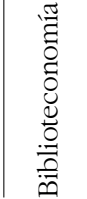 & 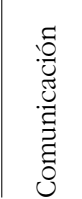 & 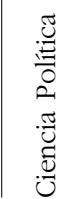 & 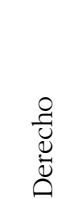 & 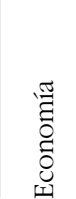 & 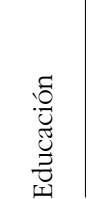 & 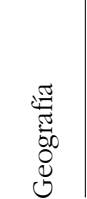 & $\begin{array}{l}\frac{\pi}{0} \\
\frac{0}{0} \\
\frac{0}{\infty} \\
0\end{array}$ & $\begin{array}{l}\frac{80}{00} \\
0 \\
0 \\
0 \\
0 \\
0\end{array}$ & 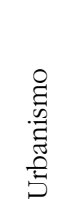 \\
\hline
\end{tabular}




\section{FIGURA 3}

Distribución de diferentes indicadores del personal investigador según sexo. Período 2001-2008 en Derecho y 1996-2008 en el resto de disciplinas

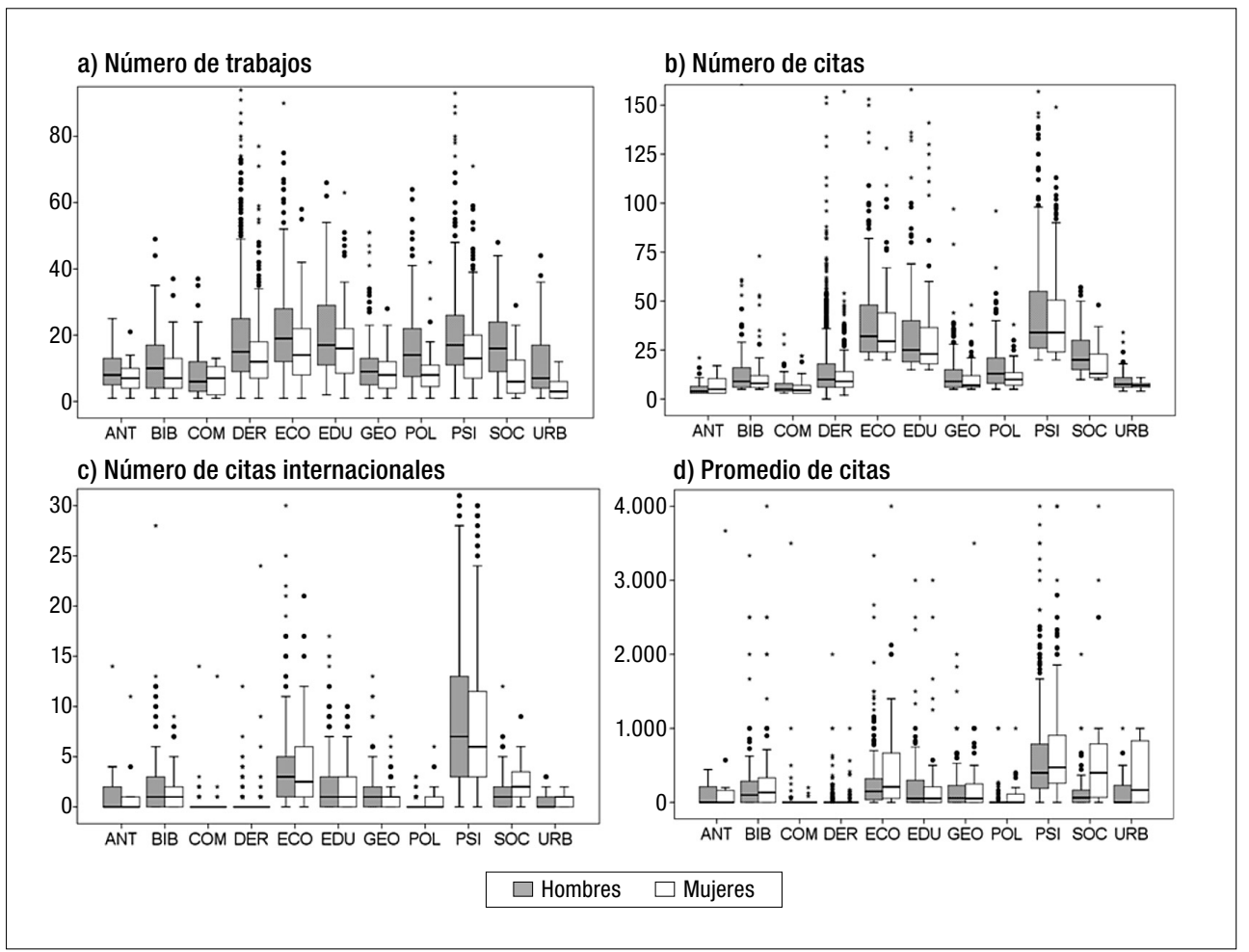

Abreviaturas: ANT-Antropología, BIB-Biblioteconomía y Documentación, COM-Comunicación, DER-Derecho, ECO-Economía, EDU-Educación, GEO-Geografía, POL-Ciencia Política y de la Administración, PSI-Psicología, SOC-Sociología, URB-Urbanismo.

\subsection{Presencia de las mujeres en la élite}

Para examinar la presencia de las mujeres en la élite científica, entendida ésta como el personal investigador que alcanza las puntuaciones más altas en los indicadores bibliométricos, hemos analizado cuál es el porcentaje de mujeres presentes en el primer tercio de los rankings INRECS/INRECJ atendiendo a los diferentes indicadores empleados; dicho porcentaje se compara con los obtenidos previamente en la tabla I. En principio en una situación científica de igualdad sí la cuota de investigadoras, teniendo como referencia los datos del INE, representa un porcentaje determinado dicho porcentaje debería ser igual en el ranking de INRECS/INRECJ. Sin embargo la mayor parte de los indicadores y disciplinas revelan que las mujeres están representadas en menor medida que los hombres (figura 4). 
FIGURA 4

Presencia de las mujeres en los rankings INRECS/INRECJ comparada con las mujeres trabajando en el área científica. Período 2001-2008 en Derecho y 1996-2008 en el resto de disciplinas

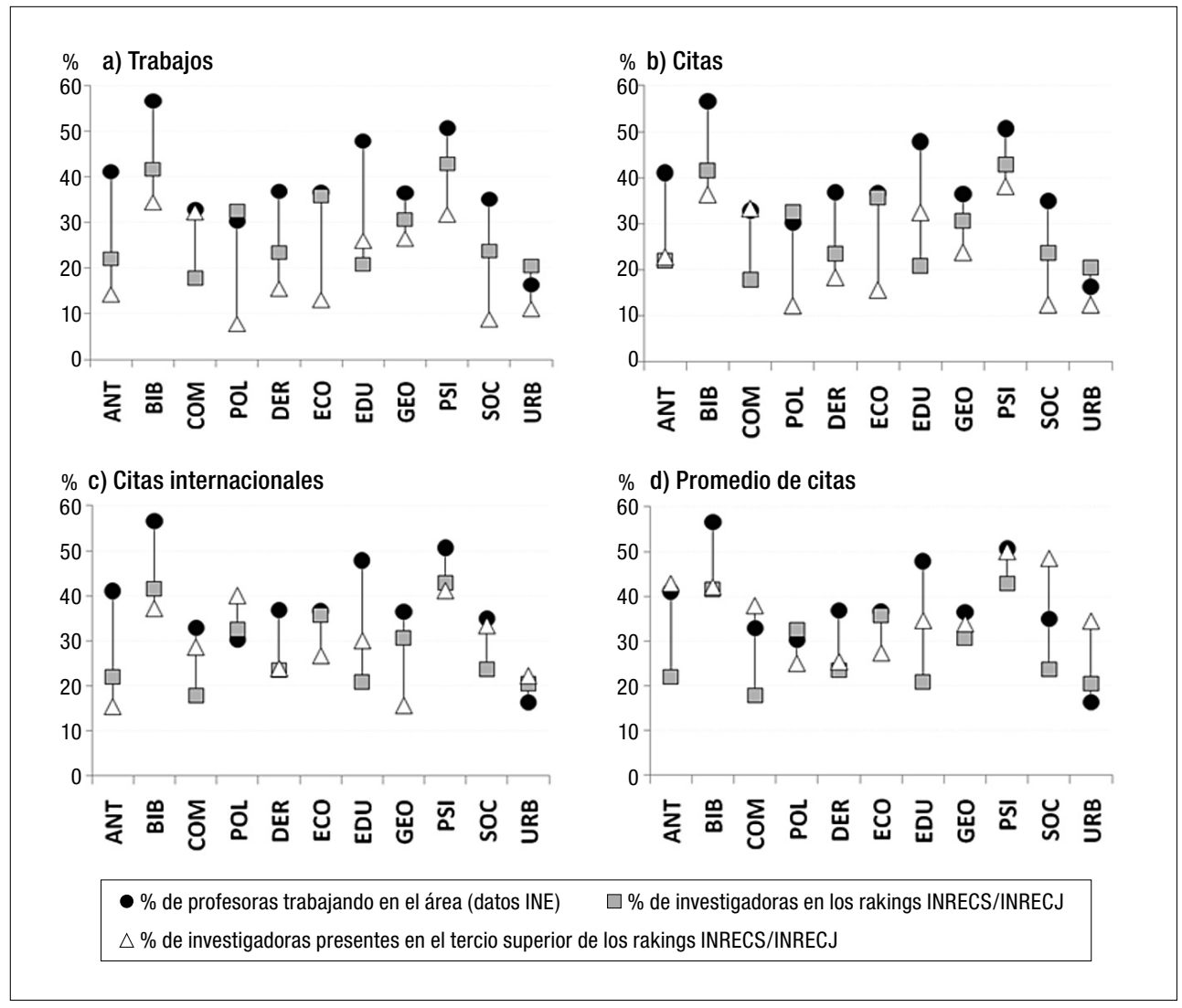

En primer lugar, si analizamos el porcentaje de mujeres en los rankings INRECS/INRECJ hay que mencionar como en la mayoría de indicadores y disciplinas no consigue superar al porcentaje de las mismas trabajando en el área (datos INE). En cuanto a la élite, en el caso de los trabajos, existen diferencias importantes entre el número de mujeres trabajando y aquellas que consiguen llegar a posiciones altas de los rankings; en áreas como Ciencia Política o Sociología las mujeres en la élite apenas alcanzan el 10\%, y es donde, precisamente, casi el $40 \%$ del personal investigador es femenino. El área donde es menos acusada esta diferencia es Comunicación. Para las citas, tanto las globales como las internacionales, vuelve a ocurrir lo mismo con diferencias significativas en las áreas de Derecho, Economía y Sociología para las citas globales y para las de Antropología, Geografía y Educación para las internacionales. Finalmente, para el promedio 


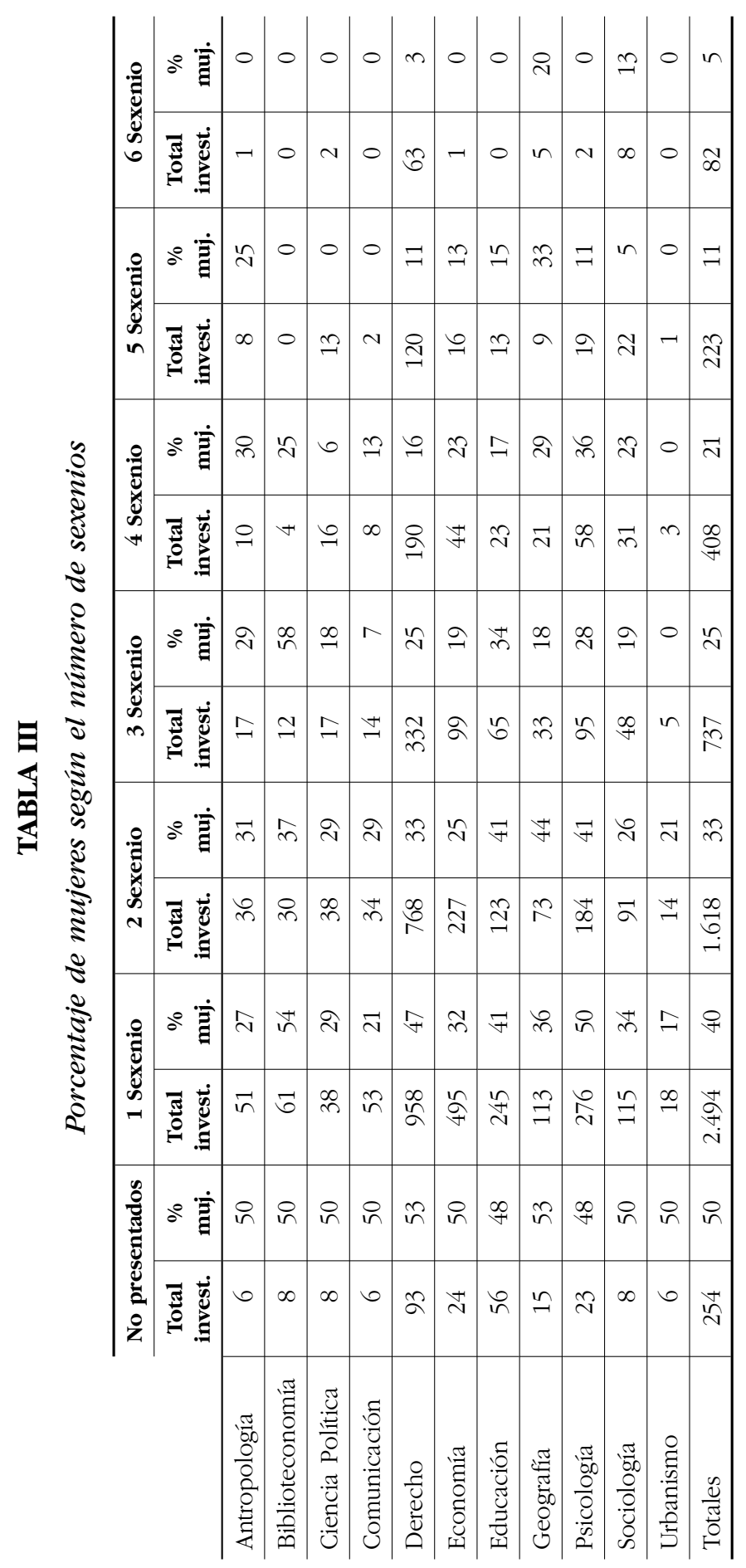


de citas sí existe un mayor equilibrio entre los diferentes porcentajes estudiados; en Antropología, Comunicación, Geografía y Psicología el porcentaje en la élite es similar al de la disciplina, y en el caso de Sociología y Urbanismo y Ordenación del Territorio, es mayor.

Otro indicador que nos puede ayudar a comprender la presencia femenina en la élite de la investigación es el porcentaje de mujeres con un determinado número de sexenios y que se presenta en la tabla III. En el total general existe una tendencia clara; conforme consideramos un mayor número de sexenios menor es el porcentaje de mujeres. Así el 40\% del personal investigador con un sexenio son mujeres y esta cifra se reduce hasta un 5\% cuando llegamos a los seis sexenios. Es relevante también que en el caso del personal que no se ha presentado nunca a evaluación la cifra sea igual para ambos sexos. Por disciplinas las investigadoras nunca representan más del 50\% en ningún número de sexenios, exceptuando el caso de 1 y 3 sexenios en Bibliotecononía, donde un $58 \%$ son mujeres.

\section{Discusión y conclusiones}

La representación global de las investigadoras en las áreas de Ciencias Sociales y Jurídicas, tomando como marco de referencia las estadísticas del profesorado universitario del INE, en relación a los investigadores está casi equiparada, existiendo disciplinas donde la mujeres representan más del el 50\% (Biblioteconomía y Psicología). Sin embargo existen diferencias significativas en la capacidad de producción de trabajos, ya que las mujeres en todas las áreas analizadas presentan un menor número de trabajos que los hombres. Esta situación en las Ciencias Sociales españolas es similar a la señalada en estudios anteriores (Cole, 1984; Schiebinger, 1993; Prpic, 1996; Valian, 1999; Kaplan y otros, 1996; Tower y otros, 2007). Esta menor productividad, que es un fenómeno que se produce también en otras áreas y países, se ha tratado de explicar a través de diferentes factores socioculturales como la responsabilidad en las cargas familiares (Kyvik y Teigen, 1996; Xie y Shauman, 1999; Rothausen-Vange y otros, 2005) o factores más relacionados con el entorno académico como los apuntados por Webster (2001) según el cual las mujeres dedican mayor tiempo a la docencia comparada con los hombres. Aunque encontrar el factor determinante que explique porqué se producen éstas diferencias de género en la productividad científica es, como diría Cole y Zuckerman (1984), un puzle aún sin resolver.

En el caso del número de citas también las mujeres obtienen valores inferiores y sólo en dos áreas consiguen superar a los hombres. Sin embargo, esta situación se invierte en el caso de las citas internacionales, ya que en el 64\% de las áreas analizadas las mujeres presentan valores superiores. El promedio de citas alcanzado por las mujeres es mayor en todas las áreas comparadas con el promedio de hombres, exceptuando el área de Biblioteconomía. Parece, por tanto, que el perfil de las investigadoras españolas está orientado a producir me- 
nos que sus homólogos masculinos. Este hecho provoca que reciban un número menor de citas brutas al publicar menos artículos; sin embargo al dividir las citas entre trabajos presentan, a nivel general, un mayor promedio de citas, por tanto publican menos pero con mayor tasa de citación. Estos resultados también están en consonancia con otros análisis previos; algunos estudios muestran que los trabajos publicados por las investigadoras son citados con mayor frecuencia que los trabajos de los hombres (Schiebinger, 1999; Sonnert y Holton, 1996; Zuckerman, 1987; Nilsson, 1997; Feller, 2004; Tower y otros, 2007; Prpic y otros, 2009) e indican que la menor productividad femenina se asocia a documentos de mayor calidad (Long, 1992). Por tanto, parece que las Ciencias Sociales y Jurídicas españolas mantienen patrones ya establecidos en otras áreas y países.

Por último, los resultados manifiestan que las investigadoras españolas están todavía en los estratos inferiores de la pirámide científica nacional, tanto en lo que se refiere a la producción como a las citas totales alcanzando la élite científica en porcentajes muy reducidos. Esta situación se evidenció porque el porcentaje de investigadoras trabajando (datos INE por área) no se equipara con el porcentaje de mujeres presentes en los rankings INRECS/INRECJ ni con el porcentaje de mujeres en el primer tercio de dichos rankings, donde en líneas generales están representadas en porcentajes muy inferiores. Existe una infrarepresentación de la mujer en las élites científicas si tenemos en cuenta la producción y citación, situación que se confirma si comparamos el porcentaje de mujeres y hombres con un mayor número de sexenios, ya que tan solo el $20 \%$ de las investigadoras cuenta con tres o más tramos de investigación. Porqué las mujeres no están equiparadas, en lo que respecta a la élite científica, se puede explicar por la incorporación tardía de la mujer a la ciencia española. Indicadores como la producción, el número de citas y, especialmente, el número de sexenios están muy ligados a la longitud cronológica de las carreras científicas. Hay que tener presente que el acceso "regularizado" de las mujeres a la carrera universitaria fue a partir de 1975, período de la transición política española. Al tener las mujeres una vida académica más corta que los hombres aún no ha transcurrido el tiempo suficiente para que éstas se posicionen en los rankings, ni para alcanzar un número significativo de tramos. Finalmente indicar que los resultados presentados en este estudio contribuyen a dilucidar con datos empíricos, por primera vez, la situación actual de las mujeres en las Ciencias Sociales y Jurídicas españolas y pueden ayudar a fijar un punto de partida y referencia para estudios futuros.

\section{Bibliografía}

Abramo, G.; D'angelo, C. A., y Caprasecca, A. (2009). The contribution of star scientists to overall sex differences in research productivity. Scientometrics, vol. 81 (1), 137-156.

Alcalá Cortijo, P.; Bordons, M.; García de Cortázar, M. L.; Griñón, M.; Guil, A.; Muñoz, A.; Pérez Sedeño, E., y Santesmases, M. J. (2005). Mujer y ciencia: La situación de las mujeres investigadoras en el sistema español de ciencia y tecnología. Madrid, Fundación Española para la Ciencia y la Tecnología. 
Arranz, F. (2004). Las mujeres y la universidad española: estructura de dominación y disposiciones feminizadas en el profesorado universitario. Politica y sociedad, vol. 41 (2), 223-242.

Bordons, M.; Morillo, F.; Fernández, M. T., y Gómez, I. (2003). One step further in the production of bibliometric indicators at the micro level: Differences by gender and professional category of scientists. Scientometrics, vol. 57 (2), 159-173.

Cole, J., y Zuckerman, H. (1984). The Productivity Puzzle: Persistence and Change in Patterns of Publication of Men and Women Scientists. En: Steinkamp, M., y Maehr, M. (eds.). Advances in Motivation and Achievement, vol. 2. Greenwich CT; Jai Press, 217-258.

Delgado López-Cózar, E.; Jiménez Contreras, E.; Ruiz Pérez, R.; López Herrera, A.; Gacto Colorado, M. J.; Torres Salinas, D.; Moneda-Corrochano, M.; Ruiz-Baños, R.; Pérez Ortega, J. M.; Bailón-Moreno, R.; Poyatos Huertas, E., y Rodríguez Gálvez, M. J. (2005). INRECS: Índice de impacto de las revistas españolas de ciencias sociales. Biblio $3 \mathrm{~W}$, Revista Bibliográfica de Geografía y Ciencias Sociales, vol. 10 (30).

CNEAI (2009). Informe 2009 sobre los resultados de las evaluaciones de la CNEAI [consulta: 03-05-2010]. Disponible en: http://www.educacion.es/horizontales/ministerio/ organismos/cneai/memorias-informes.html.

European Commission (2000). ETAN Report: Science policies in the European Union: Promoting excellence through mainstreaming gender equality. Luxembourg; European Commission. ETAN Expert Working group on Women and Science [consulta: 27-012010]. Disponible en: http://www.amit-es.org/etan.htm.

European Commission (2006). She figures, 2006: Women and Science: Statistics and Indicators. Luxemburgo; Comisión Europea, Dirección General de Investigación [consulta: 15-02-2012]. Disponible en: http://kif.nbi.dk/She_Figures_2006.pdf.

European Commission (2009). Women in science and technology-Creating sustainable careers. Luxembourg; Office for Official Publications of the European Communities.

Feller, I. (2004). Measurement of scientific performance and gender bias. En: Gender and Excellence in the Making. Luxembourg; Office for Official Publications of the European Communities, 35-40.

Fernández Esquinas, M.; Pérez Yruela, M., y Merchán Hernández, C. (2006). El sistema de incentivos y recompensa en la ciencia pública española. En: Sebastián, J., y Muñoz, E. (eds.). Radiografía de la Investigación en España. Madrid: Biblioteca Nueva, 148-206.

García de Cortázar, M. L., y Arranz, F., del Val, C.(2006). Mujeres y hombres en la ciencia española. Una investigación empirica. Madrid: Instituto de la Mujer.

García de León, M. A., y García de Cortázar, M. L. (coord.) (2001). Las académicas (profesorado universitario y género). Madrid: Instituto de la Mujer.

Gupta, B. M.; Kumar, S., y Aggarwall, B. S. (1999). A comparision of productivity of male and female scientists of CSIR. Scientometrics, vol. 45 (2), 269-289.

Guerrero-Bote, V. P.; Gómez-Crisóstomo, R.; Romo-Fernández, L. M., y Moya-Anegón, F. (2009). Visibility and responsibility of women in research papers through the order of signatures: the case of the University of Extremadura, 1990-2005. Scientometrics, vol. 81 (1), 225-238.

Huyer, S.; Westholm, G. (2002). Toolkit on Gender Indicators in Engineering, Science and Technology. UNESCO/GAB, París: Unesco [consulta: 27-01-2010]. Disponible en: http:// gstgateway.wigsat.org/toolkit/ch1.html. 
Instituto Nacional de Estadística (2010). Estadística para la Enseñanza Universitaria en España, curso 2007-2008: Personal docente de los centros propios de las Universidades Públicas por Área de conocimiento, Sexo y Categoría [consulta: 26-03-2010]. Disponible en: http://www.ine.es/.

Jiménez-Contreras, E.; Delgado López-Cózar, E., y Moneda Corrochano, M. (2008). The impact of spanish social sciences as seen through the spanish research Journal. Excellence and Emergence. A New Challenge for the Combination of Quantitative and Qualitative Approaches. 10th International Conference on Science and Technology Indicators. Viena, 17-20 de septiembre. Viena, Austrian Research Centers GmbH.

Kaplan, S. H.; Sullivan, L. M.; Dukes, K. A.; Phillips, C. F.; Kelch, R. P.. y Schaller, J. G. (1996). Sex differences in academic advancement: results of a national study of pediatricians. The New England Journal of Medicine, vol. 335 (17), 1282-1290.

Kyvik, S., y Teigen, M. (1996). Child care, research collaboration, and gender differences in scientific productivity. Science, Technology \& Human Values, vol. 21 (1), 54-71.

Lewison, G. (2001). The quantity and quality of female researchers: a bibliometric study of Iceland. Scientometrics, vol. 52 (1), 29-43.

Long, J. S. (1992). Measures of sex differences in scientific productivity. Social Forces, vol. 71 (1), 159-178.

Mauleón, E., y Bordons, M. (2006). Productivity, impact and publication habits by gender in the area of Materials Science. Scientometrics, vol. 66 (1), 199-218.

Muñoz-Muñoz, A. M. (2006). Presencia y producción científica de las profesoras en la Universidad de Granada (1975-1990). Granada: Universidad de Granada, Servicio de Publicaciones.

Muñoz-Muñoz, A. M. (2005). The Scholarly Transition of Female Academics at the University of Granada (1975-1990). Scientometrics, vol. 64, (3), 225-250.

Nilsson, J. (1997). Utvärdering av de vetenskapliga rapporterna (Evaluation of the scientific reports). MFR Informerar (Information from the Swedish Medical Research Council), vol. 3, 8-9.

Palomba, R., y Menniti, A. (2001). Minerva's daughters. Rome: Institute for research on population and social policies.

Prpic, K. (1996). Scientific fields and eminent scientists' productivity patterns and factors. Scientometrics, vol. 37 (3), 445-471.

Prpic, K. (2002). Gender and productivity differentials in science. Scientometrics, vol. 55 (1), 27-58.

Prpic, K.; Suljok, A., y Petrovic, N. (2009). Gender differences in the research productivity of natural and social scientists. En: Prpic, K.; Oliveira, L., y Hemlin, S. (eds.). Women in Science and Technology. Institute for Social Research Zagreb, Zagreb.

Rothausen-Vange, T. J.; Marler, J. H., y Wright, P. M. (2005). Research Productivity, Gender, Family, and Tenure in Organization Science Careers. Sex Roles, vol. 53 (9-10), 727-738.

Ruíz-Pérez, R.; Delgado López-Cózar, E., y Jiménez-Contreras, E. (2003). Spanish name indexing errors in international databases. The Lancet, vol. 361 (9369), 1656-1657.

Russell, J. M. (2003). Los indicadores de producción científica por género: Un caso especial. Tercer Taller de Obtención de Indicadores Bibliométricos, Red Iberoamericana de Indicadores de Ciencia y Tecnología (RECYT) y el Centro de Información y Docu- 
mentación Científica (CINDOC) de España, Madrid, 3 al 5 de marzo [consulta: 27-012010]. Disponible en: http://www.ricyt.org/interior/normalizacion/III_bib/Rusell.pdf.

Schiebinger, L. (1993). Nature's Body: Gender in the Making of Modern Science. Boston: Beacon Press.

Schiebinger, L. (1999). Has Feminism Changed Science? Cambridge: Harvard University Press.

Sonnert, G., y Holton, G. (1996). Career patterns of women and men in the sciences. American Scientist, vol. 84 (1), 63-71.

Tower, G.; Plummer, J., y Ridgewell, B. (2007). A multidisciplinary study of gender-based research productivity in the world's best journals. Journal of Diversity Management, vol. 2 (4), 23-32.

Valian, V. (1999). Why So Slow?. The Advancement of Women. MIT press.

Webster, B. M. (2001). Polish women in science: a bibliometric analysis of Polish science and its publications. 1980-1999. Research Evaluation, vol. 10 (3), 185-194.

Xie, Y., y Shauman, K. (1999). Commentary: Gender Differences in Research Productivity. The Scientist, vol. 13 (19), 10.

Zuckerman, H. (1987). Persistence and change in the careers of men and women scientists and engineers: A review of current research', En: Dixon, L. S. (ed.). Women: Their Under-Representation and Career Differentials in Science and Engineering. Washington; National Technical Information Service, 123-156. 